\title{
Prognostic Significance of Tumor-infiltrating Lymphocytes and Androgen Receptors in Patients with Early Triple-negative Breast Cancer
}

\section{Ana Tečić Vuger ( $\square$ ana.tecic.vuger@gmail.com )}

University Hospital for Tumors, Sisters of Charity University Hospital Center

\section{Robert Šeparović}

University Hospital for Tumors, Sisters of Charity University Hospital Center

\section{Sanda Šitić}

University Hospital for Tumors, Sisters of Charity University Hospital Center

\section{Ljubica Vazdar}

University Hospital for Tumors, Sisters of Charity University Hospital Center

\section{Mirjana Pavlović}

University Hospital for Tumors, Sisters of Charity University Hospital Center

\section{Damir Vrbanec}

Juraj Dobrila University of Pula

\section{Research Article}

Keywords: early breast cancer, triple negative breast cancer, tumor-infiltrating lymphocytes, central tumor, invasive margin, androgen receptors, biomarkers

Posted Date: September 27th, 2021

DOI: https://doi.org/10.21203/rs.3.rs-927413/v1

License: (c) (1) This work is licensed under a Creative Commons Attribution 4.0 International License. Read Full License 


\section{Abstract}

\section{Aims}

This study aimed to investigate the association between tumor-infiltrating lymphocytes (TIL) and androgen receptors (AR) and to assess their impact on early triple-negative breast cancer (TNBC) prognosis. Previous studies analyzed only stromal TIL (sTIL) and intratumoral (ITIL), while this study includes an additional spatial analysis for TIL in central tumor (CT) and invasive margin (IM) compartments and correlation with AR expression and overall survival (OS).

\section{Methods}

A retrospective cohort study encompassing 152 early TNBC tissue samples from patients treated at a tertiary oncologic center between 2009 and 2012. TIL and AR were assessed using formalin-fixed paraffin-embedded samples, using hematoxylin-eosin staining and immunohistochemistry. AR-positive tumors were considered those with $\geq 1 \%$ nuclear-stained cells.

\section{Results}

High TIL indicators were found to be positive prognostic factors. Although AR was not an independent prognostic factor, its interactions with sTIL and ITIL at IM impacted OS. Positive AR along with high sTIL and ITIL in IM were associated with favorable OS (HR for sTIL $0.22 ; 95 \% \mathrm{Cl} 0.05-0.97 ; \mathrm{p}=0.045$ and HR for ITIL 0.10; $95 \% \mathrm{Cl} 0.01-0.78 ; \mathrm{p}=0.028$ ).

\section{Conclusion}

Spatial morphological analysis of TIL reveals an additional prognostic value when combined with AR status, and shows a clinically significant impact on OS in early TNBC.

\section{Introduction}

Triple-negative breast cancer (TNBC) is responsible for one-sixth of all breast cancer cases, associated with highly aggressive behavior and a poor prognosis. $(1,2)$ It represents a subset of patients marked by heterogeneiety, with specific molecular characteristics that portend early relapse and rapid progression of disease. $(3,4)$ In the absence of a valid biomarker, and thus a tool to monitor targeted treatment outcomes, chemotherapy remains the backbone of TNBC treatment. TNBC exhibit a paradox in treatment - better chemosensitivity and response to therapy, but worse survival. (5) Modern research aims put an emphasis on finding new biomarkers that will be predictive and prognostic in TNBC, such as androgen receptors (AR) and tumor-infiltrating lymphocytes (TIL). Little is known about the association of TIL and AR in TNBC, especially about their joint effect on the prognosis in early disease stages. Several 
monoinstitutional analyses on a small number of patients in the last three years have shown that there is generally no statistically significant correlation between these parameters. $(6,7,8)$ On the other hand, analyses on TIL to date have evaluated mostly stromal and intratumoral TIL, leaving room to reconsider the connections between TIL and AR from a spatial analysis standpoint. If these variables are considered separately in compartments of central tumor (CT) and invasive margin (IM), additional information related to prognosis may be uncovered. The aim of this study was to investigate the association of spatial STIL and ITIL analysis in the CT and IM tumor compartments and AR in early TNBC, and their prognostic significance.

\section{Results}

We enrolled 152 patients with the median (IQR) age 58 (47-70) years, 64\% were postmenopausal, $84 \%$ diagnosed with NOS, with median (IQR) size of tumor of $2.2(1.55-2.95) \mathrm{cm}, 41 \%$ of them with positive lymph nodes, $22 \%$ in stage I, $59 \%$ in stage II, and $19 \%$ in stage III of disease. Radical mastectomy was performed in $39 \%$ of patients, $88 \%$ were treated with adjuvant chemotherapy and $74 \%$ with adjuvant radiotherapy. No patients were treated with the neoadjuvant approach.

Positive AR defined as tumors with $\geq 1 \%$ nuclear-stained cells, was present in 47 (31\%) patients, and AR defined as tumors with $\geq 10 \%$ nuclear-stained cells, in $40(26 \%)$ patients. Distribution of AR expression was markedly positively asymmetric and statistically significantly different from the normal distribution. (Shapiro-Wilk test, $n=151, W=0,83 ; V=20,07 ; z=6,8 ; p<0.001$ ). Similarly, presence of TIL in spatial analysis showed a positive asymmetric distribution. Spatial morphological analysis of TIL by compartments revealed that: median STIL was $19 \%$, median TTIL 5\%, median TIL in CT 5\%, and median TIL at IM 18\%; median STIL in CT 5\%, median ITIL in CT 1\%, median STIL at IM 30\%, and median iTIL at IM 5\%. Prevalence of intermediate or high TIL expressions defined in accordance with the International TIL Working Group criteria as $\geq 10 \%$ were: sTIL in CT in $48 \%$ of cases, iTIL in CT in $23 \%$, sTIL at IM in $86 \%$, and $\mathrm{TTIL}$ at IM in $47 \%$ of cases.

\section{Association of AR expression and TIL presence}

Expression of AR was not statistically significantly associated with the presence of sTIL and iTIL, or with TIL in individual compartments (Table 1; Fig. 1). 
Table 1

Association of AR expression and TIL presence by compartments

\begin{tabular}{|llll|}
\hline \multicolumn{5}{|c|}{ AR } & & \\
\hline & $\rho$ & $(95 \% \mathrm{Cl})$ & 0 \\
\hline STIL & -0.04 & $(-0.21-0.12)$ & 0.598 \\
iTIL & -0.05 & $(-0.21-0.10)$ & 0.516 \\
\hline STIL CT & -0.06 & $(-0.22-0.10)$ & 0.463 \\
\hline iTIL CT & -0.08 & $(-0.24-0.09)$ & 0.349 \\
sTIL IM & -0.05 & $(-0.21-0.11)$ & 0.549 \\
\hline iTIL IM & -0.07 & $(-0.23-0.09)$ & 0.403 \\
\hline$\rho=$ Spearman's range correlation coefficient; Cl = confidence interval; \\
\hline p = statistical significance of correlation coefficient \\
\hline
\end{tabular}

\section{AR and TIL interaction as prognostic markers}

In univariate analysis, all TIL indicators except ITIL CT showed significant positive correlation with improved OS, while AR status did not show an impact on OS. ITIL in IM emerged as the best prognostic factor in univariate analysis. Patients with high iTIL in IM had a 5-year survival of $86.1 \%$, unlike $62.5 \%$ in patients with low ITIL in IM. When all four TIL compartments, AR and all clinical and histopathological variables were included in a multivariate regression model, only high ITIL in CT was independently associated with favorable survival (Table 2). 
Table 2

Cox regression analysis showing the association between TIL indicators, AR expression and overall survival.

\begin{tabular}{|llll|}
\hline & HR & $(95 \%$ Cl) & P \\
\hline Univariate & & & \\
\hline sTIL CT & 0.97 & $(0.94-0.99)$ & 0.011 \\
\hline iTIL CT & 0.95 & $(0.90-1.00)$ & 0.053 \\
\hline STIL IM & 0.97 & $(0.95-0.99)$ & $<0.001$ \\
\hline iTIL IM & 0.93 & $(0.89-0.98)$ & 0.005 \\
\hline AR & 1.00 & $(0.99-1.01)$ & 0.821 \\
\hline Adjusted* & & 0.060 \\
\hline sTIL CT & 1.05 & $(1.00-1.11)$ & 0.039 \\
\hline iTIL CT & 0.93 & $(0.86-1.00)$ & 0.099 \\
\hline sTIL IM & 1.05 & $(0.99-1.11)$ & 0.525 \\
\hline iTIL IM & 0.97 & $(0.88-1.07)$ & 0.673 \\
\hline AR & 1.00 & $(0.99-1.02)$ & \\
\hline $\begin{array}{l}\text { HR - hazard ratio; Cl = confidence interval; *adjusted for age, comorbidities, menopausal status, PHD, } \\
\text { tumor size, N status, stage of disease, grade, Ki 67, type of surgery, adjuvant chemo }\end{array}$ \\
\hline
\end{tabular}

Although AR was not an independent prognostic factor, it's interactions with STIL and ITIL at IM impacted OS.

In a subgroup analysis, patients with high STIL at IM and positive AR had favorable outcome when compared with patients with high sTIL at IM and positive AR (HR 0.22; 95\% Cl 0.05-0.97; $\mathrm{p}=0.045)$ (Fig. 2). The same pattern was identified in patients with high iTIL at IM and positive AR (HR 0.10;95\% Cl $0.01-0.78 ; p=0.028$ ) for an adverse outcome compared to the patients with low ITIL at IM and negative AR (Fig. 3). Five-year survival rate was $70.0 \%$ in patients with low iTIL at IM and negative AR, unlike $95.1 \%$ in patients with high iTIL and positive AR. Moreover, patients with high sTIL and positive AR had a 5-year survival rate of $78.0 \%$, unlike $66.0 \%$ in patients with low sTIL and negative AR.

\section{Discussion}

The relationship between AR and TIL in breast cancer, especially in TNBC, has only recently raised interest in literature. Although the interactions between androgen signaling pathways and the immune infiltrate has been previously described in prostate and urinary bladder cancer, literature on this topic in breast cancer is scarce. $(11,12)$. One study analyzed the relationship between AR and TIL on 50 early TNBC 
patient samples, stages I to III, showing that as many as $70 \%$ of samples had a high TIL infiltration (> $80 \%$ ) and $26 \%$ were AR positive. No statistically significant association was found between AR and TIL, but CD8 was shown to be more frequently present in AR positive tumors and CD4 in AR negative tumors. $(6,9)$ AR expression was more common in tumors with low Ki-67 but, unusually, in younger patients and in $\mathrm{N}+$ disease. High TIL infiltration was more common in aggressive tumors, with high grade and Ki-67 levels, but these tumors showed lower rates of disease recurrence and death. At the same time, they reported lower levels of CD8. (6) In 2019, another analysis examined the association between AR and TIL in 36 patients with early and metastatic TNBC. AR cut-off was $\geq 1 \%$, and TIL were evaluated by HE staining with defining LPBC if sTIL $\geq 50 \%$. AR were expressed in $19.4 \%$ of patients, with a median TIL of $15 \%$ in AR + and $33 \%$ in AR-, but without statistical significance regarding TIL values. (9) On the other hand, studies on CD3 T-cell markers showed they are more common in AR+, while CD20 is more common in AR-. The CD8 prevalence was not statistically different between AR + and AR-, but CD8 was significantly less present in the more advanced stages of disease (III and IV), compared to the lower (I and II). (14) A positive correlation between CD8 and CD3 was found, but with conflicting negative associations also described between CD3/CD20 and CD4/CD8. (7) An analysis of the AR prognostic role in 139 patients with early and metastatic TNBC, compared paired cases with present/absent disease dissemination, and AR status was identifed as a prognostic indicator, especially in TNBC patients not treated with systemic antineoplastic therapy. In the group receiving systemic treatment, the best outcome was in the low AR group (1 to 34\%). The presence of STIL was also included in the analysis, but without a report of a statistically significant correlation between the two parameters (8). A link between AR and the immune infiltrate in breast cancer was made by gene expression analysis in TNBC that defined three tumor subgroups, a non-basaloid, LAR subtype with a scarce immune response in about $20 \%$ of cases, BL with a scarce immune response and M2 macrophages, in almost $50 \%$ of TNBC cases and basaloidenriched in strong immune responses and few M2 macrophages, in about $30 \%$ of cases. (15) In a study detailing AR and TIL effect on tastuzumab therapeutic response on 150 patients with metastatic HER2positive breast cancer, AR expression was present in over $80 \%$ of cases (ER were positive in over $50 \%$ of cases) and was negatively correlated with M2 TAM (Tumor Associated Macrophages), and CD8 and CD3, but only in case of ER+. (16) Overall, AR were shown to be more pronounced in samples with low immune infiltrate, and vice versa, tumors with a high percentage of immune infiltrate were more often AR negative. More importantly, although only in exploratory analyses, patients with high AR expression and high immune infiltration were found to have a statistically significantly better OS, compared with tumors with low AR expression and high immune infiltrate, whereas there were no differences in OS in the other groups. It should, however, be noted that the study also found that the correlation between AR and immune infiltrate, that is, each of the individual IHC evaluated immune cell lines, differed in dependence of ER expression. $(16,17,18,19)$, Published data suggest that at least two key independent sources of biological information in TNBC (cellular and immune, and in addition to molecular data), have generated, a new immune classification of TNBC. (20) A study by Dieci et al. also demonstrated a prognostic role of AR in the TNBC cohort, noting that AR positive tumors were significantly associated with lower TIL values. On the other hand, although AR influenced prognosis, independent of disease stage, by adding TIL as a variable to the analysis, only TIL along with stage remained a strong independent prognostic 
factor. AR positive tumors had significantly worse DDFS, compared to AR negative tumors. (21) The association between high-AR tumors and antitumor regulatory $T$ cells in general was also established, with AR-negative tumors positively correlated with antitumor immune cells, and AR positive tumors were inversely correlated, but had better survival. (22) The above results differ from those previously described in the TNBC and HER2 + populations, which once again indicates a more complex network of interactions of all these molecules defining breast cancer subtypes, and the modulatory influence of each on the correlation of the others. Finally, a recently published analysis showed that TIL in TNBC were a good prognostic indicator, that AR in TNBC were a negative prognostic indicator, that AR + tumors generally had poor TIL infiltration, and the worst DFS was in TIL- / AR + tumors. TIL in the TIL + / AR + subgroup appear to play a protective role, preventing disease recurrence and worse outcomes for these patients. (23)

No correlation of AR expression and TIL presence was observed in this analysis, however, a potential additional prognostic value was observed in the interaction of AR with TIL at the IM. The described heterogeneity of results with respect to the relationship of AR and TIL among different breast cancer subtypes could be somewhat expected, given the trend of different roles of each parameter individually in $\mathrm{ER}+, \mathrm{HER} 2+$ and TNBC. It remains to be considered how many factors affect the relationships among the investigated parameters, the impact of which has not yet been defined or quantified. Modelled on the modulatory influence of, for example, the ER on the ratio of AR and TIL, similar can be thought about other molecular parameters, especially in the TNBC category. The described relationship of two apparently unrelated parameters, the luminal $(A R+)$ subtype of tumor cells and the activation of the host intrinsic immune response, carries the potential in better defining TNBC prognosis.

The limitations of this research are associated with the retrospective model containing the possibility of systematic bias. This is an unicentric analysis, covering only patients treated for a certain period of time at a single center. Also, data such as more detailed pathohystological description, condition of margins on the postoperative sample, presence of DCIS component, LVI and PNI and some sociodemographic components such as BMI, family history and comorbidity were missing for a larger number of patients in the sample, and these variables were not included in the final analysis. In this analysis, parameters such as molecular markers for TNBC, such as CK5/6, EGFR, are missing, meaning TNBC was not categorized into basaloid, 5NP, luminal.., what certainly affects the expression of the analyzed variables in this study. Overall, the sample had a small absolute number of AR positive, especially AR highly positive patients, so we did not obtain statistical significance in the multi-parameter analyses as well as in the survival analyses for the high-AR category. However, this study is one of the first and analyses of AR and TIL in a homogeneous cohort of patients with early TNBC using spatial TIL analysis. The associations between AR and TIL were extremely low $(\rho<0.08)$ and negative. Based on sample size and empirical AR distribution, reliable conclusions on whether the variables are independent are difficult, but our results give ground to an assumption that larger samples may show a positive correlation, especially after a value of 60 with sTIL at IM and 40 with iTIL at IM.

\section{Methods}


To address the aim of the study, a retrospective cohort study encompassing 152 tissue samples of early TNBC patients diagnosed and treated at a tertiary oncologic referral center between 2009 and 2012 was designed. The study was approved by the University Hospital Center Bioethical Board adhering to the Helsinki Declaration Revision of 1989 and written informed consent was obtained from all the participants. It has been assembled according to Strengthening the Reporting of Observational studies in Epidemiology (STROBE) guidelines. TIL and AR were assessed using standard FFPE histopathological tissue samples. Morphological analysis of TIL was performed in accordance with the recommendation of International Working Group for the Evaluation of TIL by HE staining, identifying STIL and ITIL separately in CT and IM. (9) AR was identified using immunohistochemistry, with the cut-off value for positivity of $\geq 1 \%$, as per current ASCO/CAP recommendations on hormone receptor (ER and PR) analysis. (10) Inclusion criteria were: 1) patients aged $18-80$ years, 2) patients with a definitive histopathological diagnosis of TNBC, 3) no prior oncological treatment, 5) no inflammatory or hematologic disorder affecting the peripheral cell count, 6) complete medical history, 7) minimum follow-up period of five years. Exclusion criteria were related to incomplete patient data and follow-up.

The primary predictor variables included heterogenous data grouped into logical sets: age, comorbidities, menopausal status, histological type of tumor, tumor size, number of positive lymph nodes, stage of disease, Ki-67 proliferation index, type of surgery of the breast and axilla, and treatment with adjuvant chemotherapy and radiotherapy. Data analysis was aimed at evaluating associations between TIL and AR values as variables and overall and disease-free survival as a primary end point. The continuous variables' distribution normality was verified by the Shapiro-Wilk test. In the case of statistically significant deviations from the theoretically expected normal distributions, they are described by the median and interquartile ranges. Bivariate association of predictors and confounding variables with the primary outcome was verified by a series of binary logistic regression analyses. All variables that were found to be statistically significantly related to the criterion at the $p<0.25$ level, were included in the multivariable, binary, logistic model. Every variable that was significantly associated with survival was further analyzed with a ROC (Receiver Operating Characteristic) analysis and a cut-off value for complication occurrence was identified using the Youden $\mathrm{J}$ index (measuring the sensitivity and specificity of a dichotomous tested variable) and patients were divided into low risk (< the cut-off value) and high risk subgroups (> the cut-off value). Associations between possible prognostic factors and overall patient survival were analysed using Kaplan-Meier survival analysis using the log-rank test. Secondary outcomes were analyzed by multivariate regression analysis with a Cox proportional hazard model and binary logistic regression. The level of two-way statistical significance was determined at the $p<0.05$ level and all the confidence intervals were calculated at the $95 \%$ level. Statistical analysis was made in StataCorp. 2019. Stata Statistical Software: Release 16. College Station, TX: StataCorp LLC.

\section{Conclusion}

No correlation of AR expression and TIL presence was observed in this analysis, but spatial morphological analysis of TIL reveals additional prognostic value when combined with AR status, and shows a clinically significant impact on OS in early triple-negative breast cancer patients. The described 
relationship of two apparently unrelated parameters, concerning tumor cells and compartment-specific activation of the host intrinsic immune response, carries possible prognostic potential.

\section{Declarations}

\section{Acknowledgements}

We wish to tank Ivan Kruljac and Solmed Group Ltd in assistance with biostatistical analysis and copyediting.

\section{Author contribution}

A.T.V. designed and carried out the study. She was involved in the interpretation of results and wrote the manuscript. M. P. and S.Š. were responsible for data acquisition and interpretation of results. R.Š., Lj.V., and D.V. contributed to the interpretation of data, drafted and revised the manuscript. All authors have approved the submitted version and have agreed to be personally accountable for their own contributions and to the integrity of the entire manuscript.

Additional information: This study did not receive any funding.

Conflict of interest: none declared.

Data availability: The majority of data used and analyzed in the current study is included in the manuscript. The remainder is available from the corresponding author upon request.

\section{References}

1. Perou, C. M. et al. Molecular portraits of human breast tumours. Nat, 406, 747-752 (2000).

2. Foulkes, W. D., Smith, I. E. \& Reis-Filho, J. S. Triple-Negative Breast Cancer. N. Engl. J. Med, 363 (20), 1938-1948 (2010).

3. Dent, R. et al. Triple-Negative Breast Cancer: Clinical Features and Patterns of Recurrence. Clin. Can. Res, 13 (15), 4429-4434 (2007).

4. Carey, L., Winer, E., Viale, G., Cameron, D. \& Gianni, L. Triple-negative breast cancer: disease entity or title of convenience? Nat. Rev. Clin. Oncol, 7 (12), 683-692 (2010).

5. Carey, L. A. et al. The Triple Negative Paradox: Primary Tumor Chemosensitivity of Breast Cancer Subtypes. Clin. Can. Res, 13 (8), 2329-2334 (2007).

6. Sánchez-Cousido, L. F. et al. Relationship between androgen receptor and tumor-infiltrating lymphocytes in triple-negative breast cancer. Ann. Oncol. 29 Suppl. 8, http://dx.doi.org/10.1093/annonc/mdy270.246 (2018).

7. Elghazawy, H. et al. Clinico-pathological relationship between androgen receptor (AR) and tumor infiltrating lymphocytes (TILs) in triple negative breast cancer (TNBC). Ann. Oncol, 30 Suppl, 3 
https://doi.org/10.1093/annonc/mdz095.070 (2019).

8. Jongen, L. et al. The prognostic role of the androgen receptor in patients with triple-negative early breast cancers and primary surgery. ASCO 37(15), https://ascopubs.org/doi/abs/10.1200/JC0.2019.37.15_suppl.e12042 (2019).

9. Hendry, S. et al. Assessing Tumor-Infiltrating Lymphocytes in Solid Tumors. Adv. Anat. Pathol, 24 (6), 311-335 (2017).

10. Hammond, M. E. H. et al. American Society of Clinical Oncology/College of American Pathologists guideline recommendations for immunohistochemical testing of estrogen and progesterone receptors in breast cancer. Arch. Pathol. Lab. Med, 134 (6), 907-922 (2010).

11. Ylitalo, E. B. et al. Subgroups of Castration-resistant Prostate Cancer Bone Metastases Defined Through an Inverse Relationship Between Androgen Receptor Activity and Immune Response. Eur. Urol, 71 (5), 776-787 (2017).

12. Tao, L. et al. Infiltrating T Cells Promote Bladder Cancer Progression via Increasing IL1, Androgen Receptor, HIF1, VEGFa Signals. Mol. Can. Ther, 15 (8), 1943-1951 (2016).

13. Denkert, C. et al. Standardized evaluation of tumor-infiltrating lymphocytes in breast cancer: results of the ring studies of the international immuno-oncology biomarker working group. Mod. Pathol, 29 (10), 1155-1164 (2016).

14. Salgado, R. et al. The evaluation of tumor-infiltrating lymphocytes (TILs) in breast cancer: recommendations by an International TILs Working Group 2014. Ann. Oncol, 26 (2), 259-271 (2015).

15. Jézéquel, P. et al. Gene-expression molecular subtyping of triple-negative breast cancer tumours: importance of immune response. Bre. Can. Res, 20, 17-43 (2015).

16. Van Rooijen, J. M. et al. Androgen receptor expression inversely correlates with immune cell infiltration in human epidermal growth factor receptor 2-positive breast cancer. Eur. J. Can, 103, 5260 (2018).

17. Lehmann, B. D. et al. Identification of human triple-negative breast cancer subtypes and preclinical models for selection of targeted therapies. J. Clin. Invest, 121 (7), 2750-2767 (2011).

18. Burstein, M. D. et al. Comprehensive genomic analysis identifies novel subtypes and targets of triplenegative breast cancer. Clin. Can. Res, 21 (7), 1688-1698 (2015).

19. Lehmann, B. D. et al. Refinement of Triple-Negative Breast Cancer Molecular Subtypes: Implications for Neoadjuvant Chemotherapy Selection. PLoS. One. 11(6), https://doi.org/10.1371/journal.pone.0157368 (2016).

20. Prado-Vázquez, G. et al. A novel approach to triple-negative breast cancer molecular classification reveals a luminal immune-positive subgroup with good prognoses. Sci. Rep, 9 (1), 1538 (2019).

21. Dieci, M. V. et al. Androgen Receptor Expression and Association With Distant Disease-Free Survival in Triple Negative Breast Cancer: Analysis of 263 Patients Treated With Standard Therapy for Stage IIII Disease. Front. Oncol, 9, https://doi.org/10.3389/fonc.2019.00452 (2019). 
22. Okano, M. et al. Estrogen Receptor Positive Breast Cancer with High Expression of Androgen Receptor has Less Cytolytic Activity and Worse Response to Neoadjuvant Chemotherapy but Better Survival. Int. J. Mol. Sci, 20 (11), 2655 (2019).

23. Mangia, A. et al. Should Tumor Infiltrating Lymphocytes, Androgen Receptor, and FOXA1 Expression Predict the Clinical Outcome in Triple Negative Breast Cancer Patients? Cancers (Basel), 11 (9), 1393 (2019).

\section{Figures}

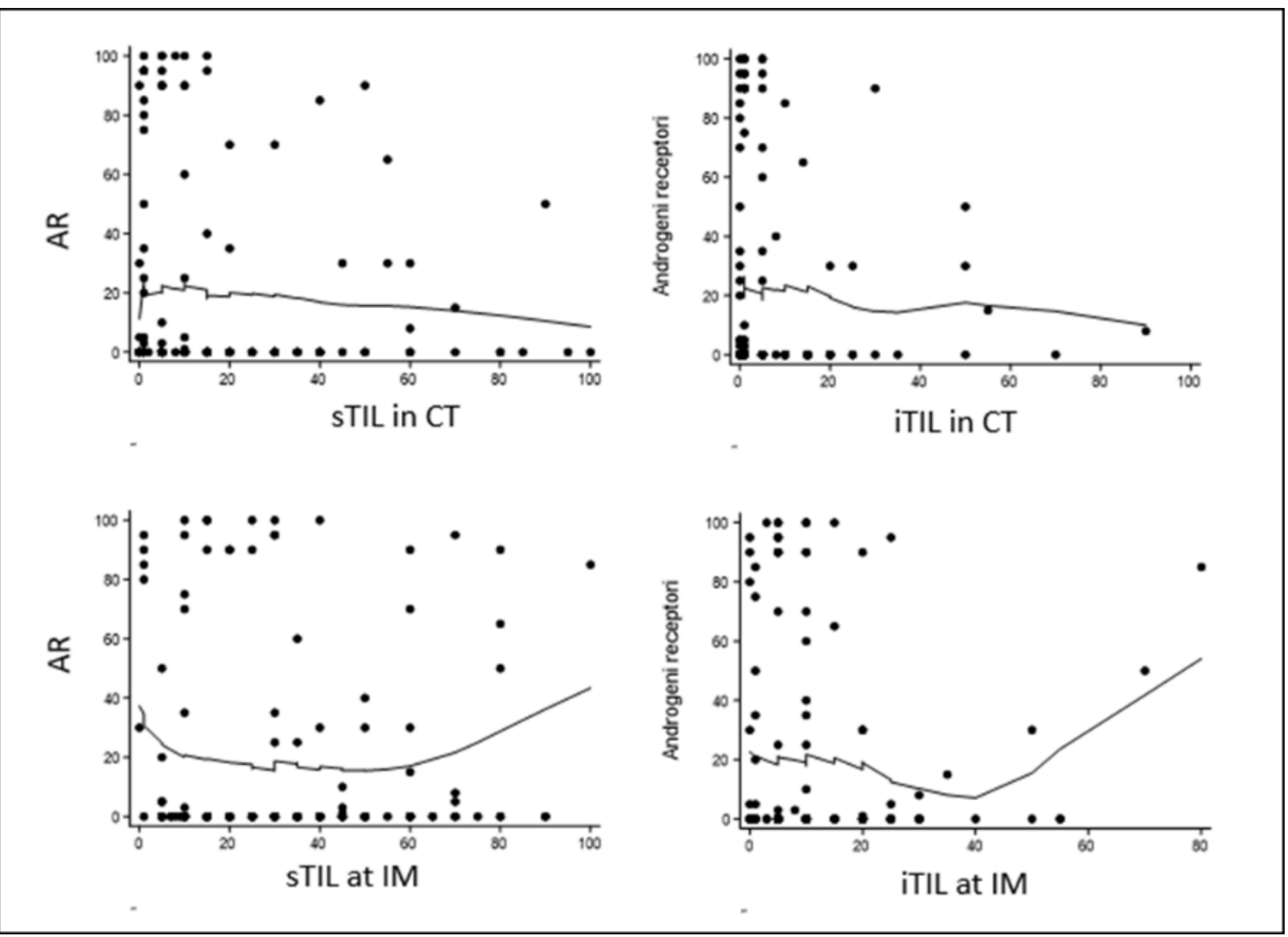

Figure 1

Point diagrams of association of AR expression with presence of TIL in individual compartments; the curve represents an $80 \%$ locally impeded polynomial regression curve 


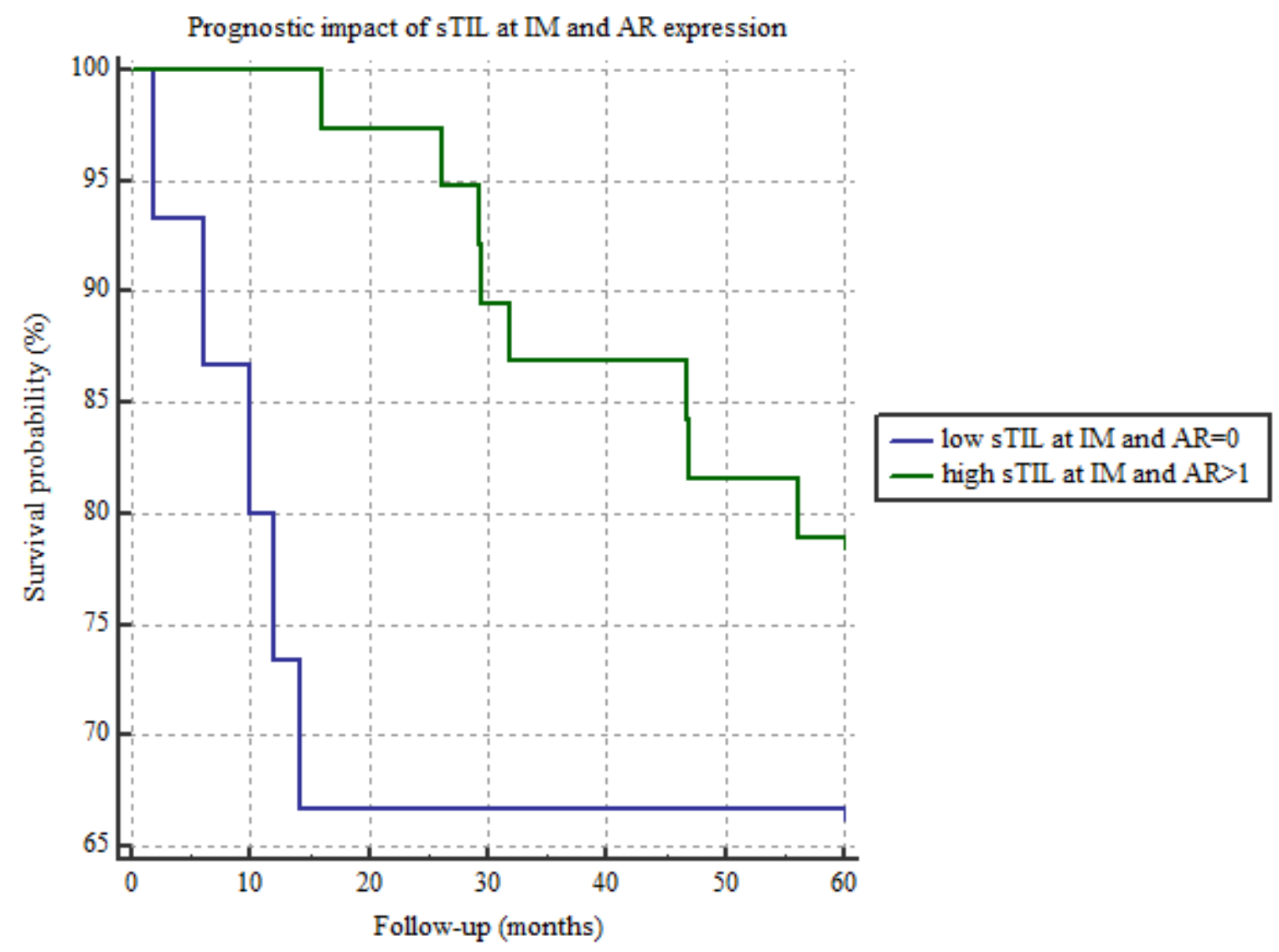

Figure 2

Kaplan-Meier curve comparing the high sTIL at IM and AR $\geq 1$ group and low sTIL at IM and AR=0 group. 


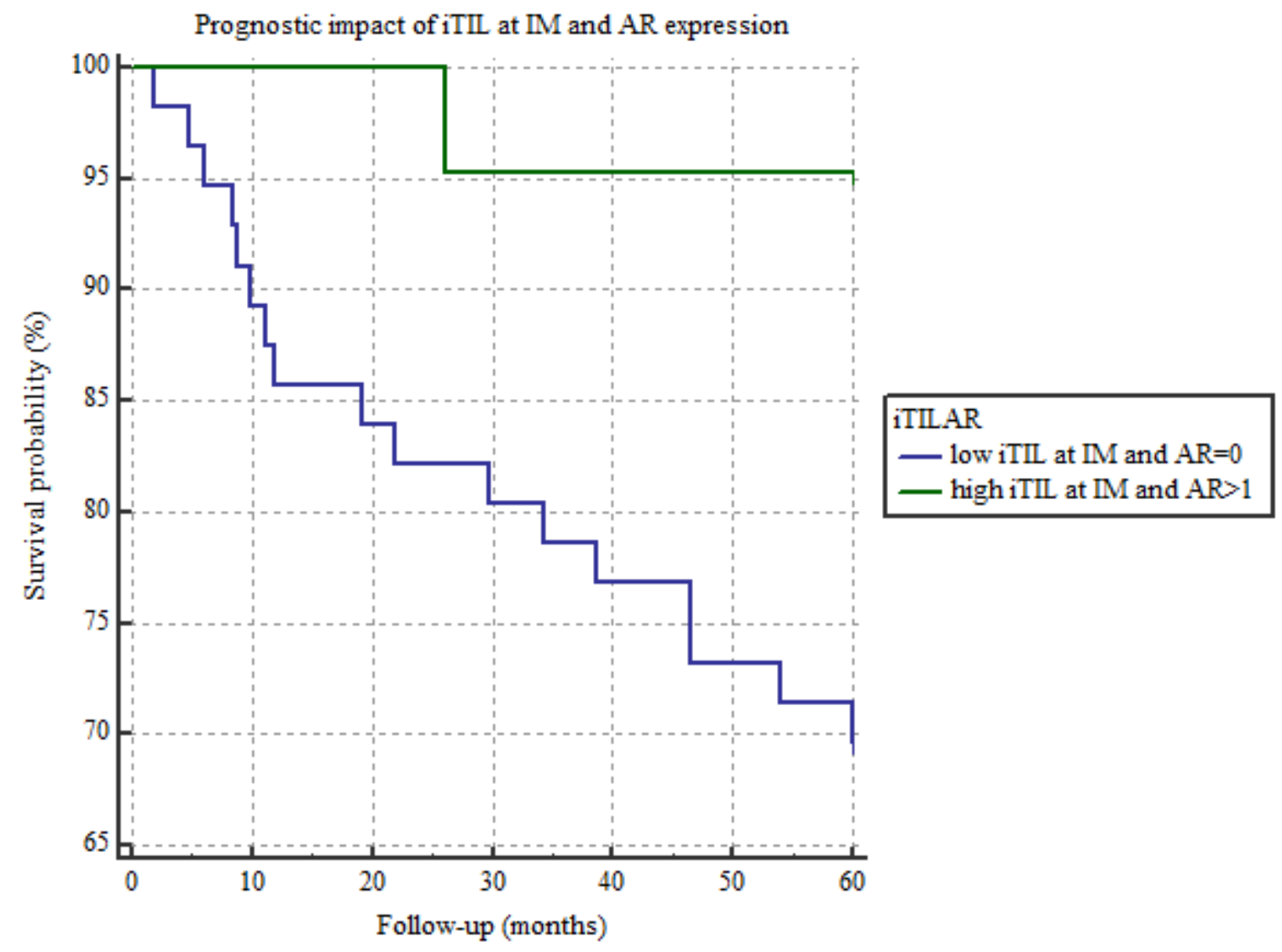

\section{Figure 3}

Kaplan-Meier survival analysis comparing the high iTIL at IM and AR $\geq 1$ group and low $\mathrm{ITIL}$ at IM and $A R=0$ group 